\title{
Fluorescence as a source of noise in Q-switched ruby laser atmospheric backscattering experiments
}

\author{
Paul D. MoCormick \\ University of Michigan, Institute of Science and Technology, \\ Haleakala Observatory (AMOS), Wailuku, Maui, Hawaii 96793. \\ (Received 25 September 1968; in revised form 25 November 1968)
}

\begin{abstract}
Interpretation of the results of atmospheric backscattering experiments, when using a $Q$-switched ruby laser as the source of photons, requires consideration of several sources of noise in the statistical analysis. This paper discusses the effects of directly backscattered ruby fluorescence in detail. It is found that, for an unshuttered system, fluorescense noise will be equal to the signal expected from altitudes above about $50-60 \mathrm{~km}$ (assuming only a molecular atmosphere). The "orossover" altitude is lowered if aerosols are present. When a shutter is used as a fluorescence block, it is found that a spurious "layer" structure may be produced-but only for shutter outoff times of greater than about $375 \mu \mathrm{sec}$. It is concluded that, for a properly designed optical radar system, ruby fluorescence will not be a significant source of spurious returns. In particular, it is concluded that this source will not explain the enhanced returns from about $80 \mathrm{~km}$ observed by MoCormrok et al. (1967).
\end{abstract}

\section{INTRODUCTION}

SEvERAL of the inherent properties of a $Q$-switched ruby laser serve to make it an almost ideal source of radiation for atmospheric backscattering experiments. Energy outputs presently available, when coupled with large telescopes, can produce detectable signals from altitudes up to about $100 \mathrm{~km}$. Pulses of the order of $10^{-8} \mathrm{sec}$ duration allow altitude resolution to be determined by the detection system or the detection statistics rather than by the radiation source. Since the radiation is almost spatially coherent the pulse can be concentrated into a relatively small volume allowing the use of a narrow receiver field of view to reduce the effects of sky background radiation. The almost temporal coherence of laser radiation permits narrow-band interference filters to be inserted in front of the detector further discriminating against sky background. It is not surprising, therefore, that many groups are now performing atmospheric backscattering experiments with $Q$-switched ruby lasers.

Due to the exponential dependence of atmospheric density upon altitude, the atmosphere can be divided into two regions from the standpoint of signal return. From below a certain altitude (about $40 \mathrm{~km}$ for typical optical radar systems) the return consists of a photoelectron current which may be displayed on an oscilloscope [NoRTHEND et al., 1966]. The signal from above this altitude is so weak that single photoelectron counting is required. Although oscilloscope photography can be used for this purpose, digital pulse counting systems are an obvious solution. An upper atmosphere optical radar system using pulse counting circuitry and an on-line digital computer has been described (McCormick et al., 1966). One group (MCConMICK et al., 1967) has reported enhanced backscattering from altitudes near 
$80 \mathrm{~km}$ while other workers have not observed such returns (BaIN and SANDFord, 1966; KeNT et al., 1967; SANDFORD, 1967). It is extremely important, therefore, to determine if the enhanced returns can be explained by a systematic source or should be ascribed to a meteorological phenomenon (e.g., accumulation of dust at the mesopause).

When interpreting the results of the upper atmosphere experiments several sources of noise must be considered. These noise sources, which are well known by those using the laser radar technique, include: photomultiplier dark current, enhanced photomultiplier noise produced by the high light levels backscattered from the lower atmosphere, sky background radiation, electronic noise, direct backscattering of ruby fluorescence and multiple scattering effects. This paper discusses the effects of backscattered ruby fluorescence in detail. In particular we examine the case in which a rotating shutter is used to block fluorescence from entering the atmosphere after the emission of the $Q$-switched laser pulse.

\section{Expected Signal due to Molecular Scattering}

Consider an upper atmosphere laser radar system with the transmitter (pulsed ruby laser and a collimating telescope) boresighted with the receiving telescope and fired toward the zenith. The detector is a photomultiplier. The return (photoelectron pulses) from each laser firing is divided into intervals of $T$ sec and the number of counts in each interval is recorded. Altitude resolution will be $c T / 2 \mathrm{~m}$ (if the laser pulse length is short compared to this value). An expression for the expected return, assuming only molecular scattering, can be written as

$$
N_{T}(z)=\frac{T Q \alpha_{r} \alpha_{a}^{2} A_{r} \lambda E_{t}}{2 h} \cdot \frac{n(z) \sigma(\pi)}{z^{2}} \text { counts per interval. }
$$

$N_{T}(z)=$ the number of photoelectron pulses counted in an interval of $T$ sec corresponding to backscattered photons originating in a layer $c T / 2$ meters thick located at an altitude $z \mathrm{~m}$.

$Q=$ the quantum efficiency of the photomultiplier for $\lambda=6943 \AA$;

$\alpha_{r}=$ the transmission of the receiver (mirrors, lenses, interference filter);

$\alpha_{a}=$ the one-way transmission of the atmosphere, for radiation of wavelength $\lambda$ meters, over a distance of $z \mathrm{~m}$;

$A_{r}=$ the effective collecting area of the receiving telescope;

$\lambda=$ wavelength of the laser output $=6943 \AA$;

$E_{t}=$ transmitted energy;

$n(z)=$ molecular concentration at an altitude $z \mathrm{~m}$;

$\sigma(\pi)=$ molecular differential backscattering cross section;

$h=$ Planck's constant;

$z=$ altitude.

A good optical radar system might have the following parameters:

$E_{t}=5 \mathrm{~J}, A_{r}=1 \mathrm{~m}^{2}, \alpha_{r}=4 \times 10^{-1}, T=10^{-5} \mathrm{sec}, Q=5 \times 10^{-2}, \theta_{t}=$ transmitter

beam width $=2.5 \times 10^{-4} \mathrm{rad}$., $\Delta \lambda=$ interference filter bandpass $=10 \AA$. 
With such a system, and if the only sources of noise were dark current and night sky radiation, molecular scattering from about $90 \mathrm{~km}$ could be measured with about 25 per cent precision in about 300 laser firings. The altitude resolution will be $1.5 \mathrm{~km}$.

\section{Expected Return from Ruby Fluorescence (No Shutter)}

Figure 1 is a very crude representation of the processes occurring when the laser is fired. The figure is not meant to represent actual pulse shapes. The flashlamps are discharged initiating excitation of the ruby and normal spontaneous emission

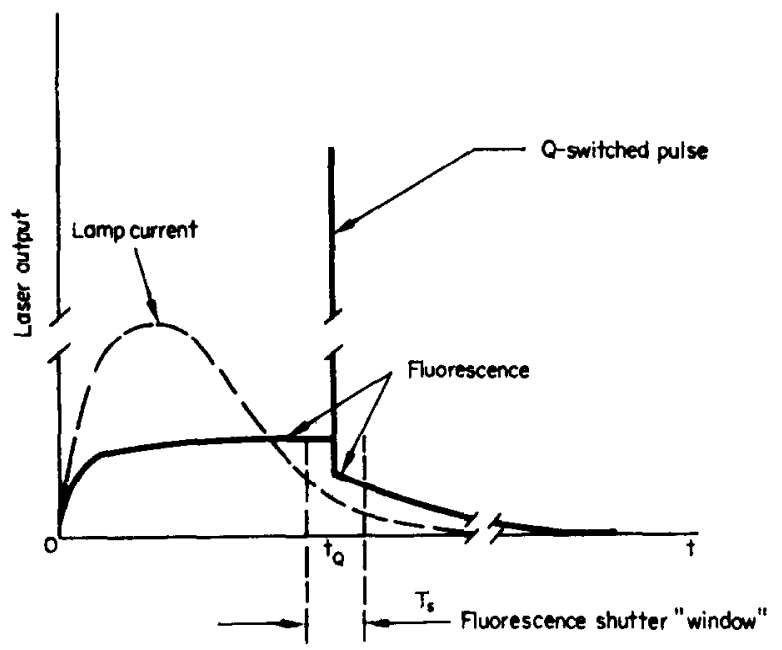

Fig. 1. Schematic of radiation emission during a laser pulse.

(fluorescence) begins to occur as shown. For $Q$-switched operation, the ruby is pumped above the lasing threshold and a resonant optical cavity is then produced (by, for example, a Pockels cell) which causes the single giant pulse to be emitted. After emission of the giant pulse the population of excited atoms is depleted below the threshold for lasing. We assume that the physical situation is such that no after-pulsing occurs. Fluorescence will, however, continue to be emitted since the flash lamps are still pumping the ruby to some extent and the spontaneous emission life-time, for ruby, is about $3 \mathrm{msec}$. The resultant effect is to cause a fluorescent tail as shown in the figure. This radiation, if not prevented from reaching the PMT, will act as a real-time background source of photons. The fluorescence could be directly scattered to the PMT by surfaces present in the system, or, if allowed to escape into the atmosphere, it will be backscattered and multiple scattered by air molecules and by particulate matter. This will result in a signal that would be falsely attributed to backscattering of the $Q$-pulse.

We make the following assumptions in order to estimate the effects of direct backscattering of fluorescence:

(a) The time origin corresponds to the emission of the $Q$-pulse. Fluorescence emitted before $t=0$ is neglected since it will be scattered from altitudes greater than that of the $Q$-pulse.

(b) Since we are interested in altitudes less than about $100 \mathrm{~km}$ we will neglect the 
exponential decay of the fluorescence. We take the fluorescence power to be constant and equal to its peak rate at $t=0$. This will allow for any flashlamp pumping that occurs after $t=0$.

(c) The fluorescence power is equal to the stored energy divided by the spontaneous emission lifetime. To calculate the stored energy we assume that the gain is just below the lasing threshold. For ruby this implies that there are about $2 N_{0} / 3$ ions in the excited state where $N_{0}$ is the number of chromium ions in the crystal.

The fluorescence power is, therefore:

$$
\begin{aligned}
P_{F} & =\frac{2 n_{i} V h c}{3 \lambda \tau} \mathrm{J} \mathrm{sec}^{-1} \\
n_{i} & =\text { number of } \mathrm{Cr} \text { ions per unit volume } \simeq 1.6 \times 10^{19} \mathrm{~cm}^{-3} ; \\
V & =\text { volume of the ruby } \\
\tau & =\text { fluorescence lifetime } \simeq 3 \times 10^{-3} \text { sec. }
\end{aligned}
$$

This radiation is essentially nondirectional. As an upper limit we assume that all of it eventually escapes from the front face of the ruby into $2 \pi \beta$ steradians where $\beta \leq 1$. (We assume that the sides of the rod and the rear mirror have a reflectivity of unity and that the Pockels cell is transparent. Neither of these assumptions will usually be true in practice. In fact, the Pockels cell is usually caused to become "opaque" a few $\mu$ sec after $t=0$ in order to prevent after-pulsing.)

The radiant intensity from the ruby is, then:

$$
J_{F}=P_{F} / 2 \pi \beta J \mathrm{sec}^{-1} \mathrm{ster}^{-1} .
$$

We now must estimate how much of this radiation can escape into the atmosphere and be backscattered toward the receiver. For this purpose we assume that the boresighted laser transmitter has a collimating telescope of diameter $d_{T} \mathbf{m}$. If the field of view of the receiver is $\theta_{r}$ radians (full angle) and the diameter of the laser rod is $d_{L} \mathrm{~m}$ then the effective angle of emission from the ruby rod will be:

$$
\theta_{L}=d_{T} d_{L}^{-1} \theta_{r} \text { rad. } \quad\left(\theta_{L}<2 \pi \beta\right) .
$$

The effective power is, then:

$$
P_{\text {eff. }}=\frac{n_{i} V h c d_{T}^{2} \theta_{r}^{2}}{12 \lambda d_{r}^{2} \tau \beta} \mathrm{J} \mathrm{sec}^{2} .
$$

At time $t$ the $Q$-switched pulse will be at an altitude $c t \mathrm{~m}$. The detector will be receiving radiation that was backscattered from an effective volume $A c \tau_{L} / 2 \mathrm{~m}^{3}$ located at an altitude $z(t)=c t / 2 \mathrm{~m}$ where $A$ is the beam cross section at $z$ and $\tau_{L}$ is the duration of the $Q$-pulse in sec.

Consider fluorescence radiation emitted at time $t^{\prime}$ in an increment of time $d t^{\prime}$. Part of this radiation will be backscattered from a volume $A^{\prime} c d t^{\prime} / 2 \mathrm{~m}^{3}$ located at an altitude $z^{\prime}=c\left(t-t^{\prime}\right) / 2 \mathrm{~m}$ and will arrive at the receiver at time $t . A^{\prime}$ is the effective beam cross section and is determined by the receiver field of view rather than by the transmitter beam divergence for the $Q$-switched pulse. 
If we assume a "pure" molecular atmosphere then an expression for the photoelectron count rate at time $t$, produced by backscattered fluorescence that was emitted at time $t^{\prime}$, can be written as:

$$
\begin{aligned}
d N_{F} & =\frac{K \exp \left(\frac{-c\left(t-t^{\prime}\right)}{2 H}\right) d t^{\prime}}{\left(t-t^{\prime}\right)^{2}} \text { photoelectrons sec }{ }^{-1} ; \\
K & =\frac{\alpha_{a}^{2} \alpha_{r} Q V A_{r} n_{0} \sigma(\pi) n_{i} \theta_{r}^{2} d_{T^{2}}^{2}}{6 c d_{L}^{2} \beta} \\
n(z) & =n_{0} \exp [-z / H] \text { molecules } \mathrm{m}^{-3} \\
n_{0} & =\text { sea level molecular concentration; } \\
H & =\text { atmospheric scale height } \simeq 8 \mathrm{~km} .
\end{aligned}
$$

The total count rate, at time $t$, is found by integrating $d N_{F}$ over the limits of $t^{\prime}$. Assuming that the transmitter and receiver beams overlap at an altitude $z_{0}$, we have an upper limit for $t^{\prime}$ of $\left(t-2 z_{0} / c\right)$ sec. The lower limit is $t^{\prime}=0$.

If we let $x^{\prime}=c\left(t-t^{\prime}\right) / 2 H$ and $c t=2 z$ we can write an expression for the total count rate, due to ruby fluorescence as a function of $z$.

$$
N_{F}(z)=\frac{c K}{2 H} \int_{z_{0} / H}^{z / H} \frac{e^{-x^{\prime}} d x^{\prime}}{x^{\prime 2}} \quad \text { counts } / \text { sec. }
$$

The integral is, to a good approximation (Appendix 1), about equal to 2 for altitudes above $48 \mathrm{~km}$. In effect, we neglect the small contribution from very high altitudes compared to the backscattering from the lower atmosphere.

We have also assumed that the beam overlap is total above $z_{0}$, zero below $z_{0}$ and $z_{0} / H \simeq \mathbf{0 . 2 5}$. This assumption will make $N_{F}$ larger since, for the system parameters assumed in this paper and a transmitter-receiver separation of about $1.5 \mathrm{~m}$, the overlap begins at $z_{0} \simeq 2 \mathrm{~km}$ and is not total until about $6 \mathrm{~km}$.

The ratio between backscattered fluorescence radiation and the return from the $Q$-switched pulse is:

$$
R_{1}(x)=K^{\prime} x^{2} e^{x} \quad(z=x H)
$$

where

$$
K^{\prime}=\frac{n_{i} V h H d_{T}^{2} \theta_{r}^{2}}{3 E_{t} d_{L}^{2} \lambda_{\tau} \beta}
$$

If we consider a ruby laser that uses a single (oscillator) rod with a volume of about $5 \times 10^{1} \mathrm{~cm}^{3}$ (this is consistent with the assumption of a $Q$-switched output of $5 \mathrm{~J}$ ) and take $d_{T} / d_{L} \simeq 20$ and $\theta_{r} \simeq 5 \times 10^{-4} \mathrm{rad}$. we can construct Fig. 2. We see that the return from backscattered fluorescence is equal to the expected molecular return at about $58 \mathrm{~km}$. At $80 \mathrm{~km}$ the ratio is about 30 and at $100 \mathrm{~km}$ it is $6 \times 10^{2}$. It should be emphasized that the altitude at which $R_{1}$ becomes unity will depend strongly on the value of $z_{0}$ for the system. For example, if $z_{0} / H=0 \cdot 1$ (beam overlap at an altitude of about $0.8 \mathrm{~km}$ ) then $R_{1}=1$ at about $50 \mathrm{~km}$. 
Although several approximations have been made it is clear that, for a reasonable and consistent set of system parameters, the atmospheric backscattering returns will be significantly contaminated by ruby fluorescence at altitudes above about $50-60 \mathrm{k}$.

If atmospheric aerosols are present the crossover altitude will be lowered. If, for example, we assume an aerosol distribution that is constant between the altitudes

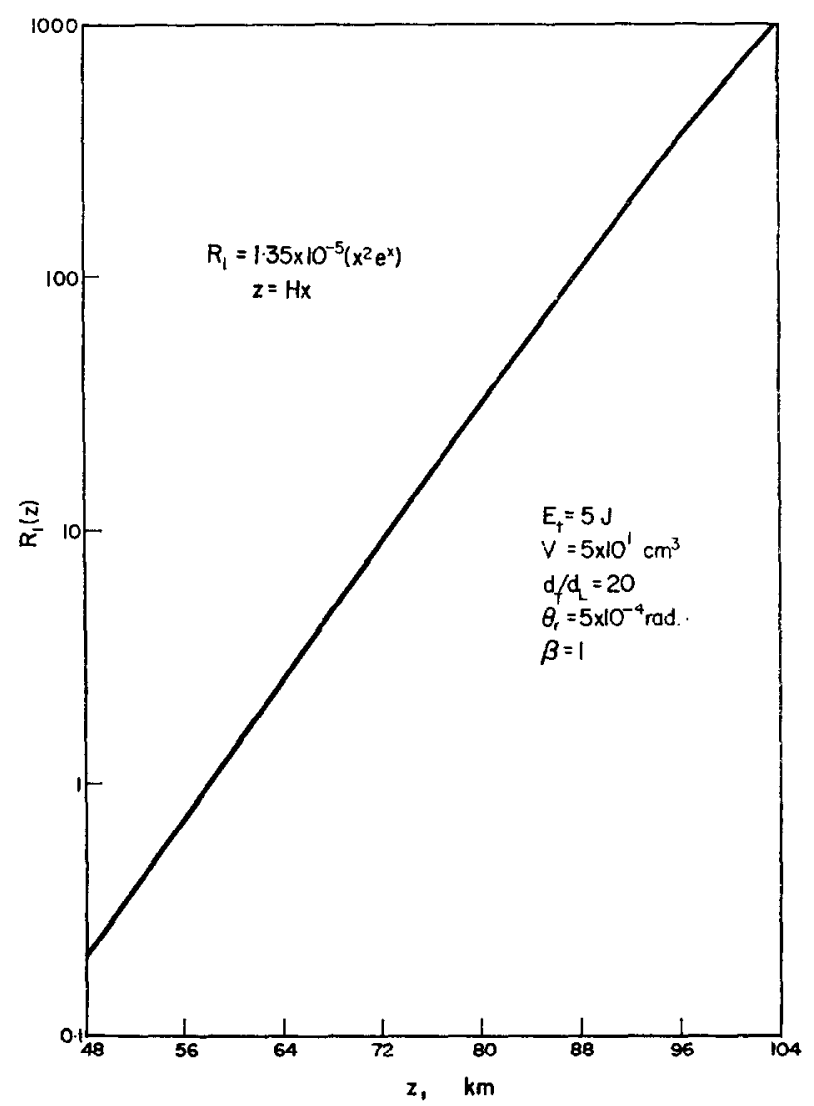

Fig. 2. Ratio of fluorescence to molecular return (no shutter).

of $z_{0}$ and $z_{0}+\Delta_{A}$ (and consists of only one type of particle) the expression for $R_{1}(x)$ will be multiplied by a factor:

$$
\begin{aligned}
& 1+\frac{\gamma_{A}}{\gamma_{M}} \cdot \frac{\Delta_{A} H}{2 z_{0}{ }^{2}} \cdot \frac{1}{1+\Delta_{A} / z_{0}} \\
& \gamma_{A}=n_{A} \sigma_{A}\left(m^{-1}\right) ; \\
& n_{A}=\text { aerosol concentration }\left(\mathrm{m}^{-3}\right) ; \\
& \sigma_{A}=\text { aerosol scattering cross section }\left(\mathrm{m}^{2}\right) ; \\
& \gamma_{m}=n_{0} \sigma(\pi) .
\end{aligned}
$$

If we take $\gamma_{A} / \gamma_{m}=3, \Delta_{A}=2 \mathrm{~km}, z_{0}=2 \mathrm{~km}$ and $H=8 \mathrm{~km}$ we find that the crossover point will occur at $x \simeq 6.25$ (compared to $x \simeq 7.25$ for the molecular case). 
For $\gamma_{A} / \gamma_{m}=100$ the crossover point is at $x \simeq 3.9$ (about $31 \mathrm{~km}$ ). Aerosols are, therefore, an important source of contamination especially for optical radars that are located at sea level and near industrial areas.

For the system parameters assumed in this paper, the combination of PMT dark current (after pulse height discrimination) and night sky background radiation would give a signal to noise ratio of unity at an altitude of about $100-110 \mathrm{~km}$. Multiple scattering, except for very unusual physical situations, would not be significant below about $150 \mathrm{~km}$ for this system. (For other systems the figure might be considerably lower.) We further assume that the PMT is protected by a shutter so that overloading does not occur. It is clear, therefore, that ruby fluorescence is the most significant source of noise in the laser atmospheric backscattering experiments.

An obvious and well-known solution to the fluorescence problem is to install a shutter in front of the ruby such that fluorescence is prevented from entering the atmosphere after the laser has been fired.

\section{Direct Backscattering of Fluorescence Passing THROUGH THE SHUTter}

Assume that the laser system is equipped with a shutter that begins to close as the $Q$-switched pulse enters the atmosphere and that fluorescence is completely blocked in $T_{s}$ sec. A tail of trailing radiation, of length $c T_{s} \mathrm{~m}$, will follow the laser pulse into the atmosphere.

If we approximate the transmission, $\alpha_{s}\left(t^{\prime}\right)$, as a linear function of time we can write:

$$
\alpha_{s}\left(t^{\prime}\right)=\left\{\begin{array}{lll}
1-t^{\prime} / T_{s} & \text { for } & 0 \leqslant t^{\prime} \leqslant T_{s} \\
0 & \text { for } & T_{s} \leqslant t^{\prime} .
\end{array}\right.
$$

The return due to the trailer pulse can be obtained by inserting $\alpha_{s}\left(x^{\prime}\right)$ into (2):

$$
N_{F}(z)=\frac{c K}{2 H} \cdot \int_{x_{0}}^{z / H} \frac{\left[1-2 H / c T_{s}\left(z / H-x^{\prime}\right)\right] e^{-x^{\prime}} d x^{\prime}}{x^{\prime 2}}
$$

$x_{0}=z_{0} / H$ or $\left(z / H-c T_{s} / 2 H\right)$ whichever is larger.

We find for the ratio of fluorescence to $Q$-pulse return, that:

$$
\begin{gathered}
R_{2}(x)=K^{\prime}\left(x^{2} e^{x}\right)\left[\left(1-\frac{x}{a}\right)\left\{\frac{e^{-(x-a)}}{(x-a)}-E_{1}(x-a)-\frac{e^{-x}}{x}+E_{1}(x)\right\}\right. \\
\left.+\frac{1}{a}\left\{E_{1}(x-a)-E_{1}(x)\right\}\right] \text { for } z_{0} / H+a \leqslant x \\
R_{2}(x) \simeq K^{\prime}\left(x^{2} e^{x}\right)\left[\left(1-\frac{x}{a}\right)\left\{2-\frac{e^{-x}}{x}+E_{1}(x)\right\}+\frac{1}{a}\left\{1-E_{1}(x)\right\}\right] \\
\text { for } z_{0} / H \leqslant x \leqslant z_{0} / I+a \\
R_{2}(x) \simeq K^{\prime}\left(\frac{a e^{a / 2}}{2}\right) \text { for } x \rightarrow \infty
\end{gathered}
$$

$\left(E_{1}(x)\right.$ is the exponential integral). 
In (4), (5) and (6) we have let $a=c T_{s} / 2 H$. Using these expressions, and the system parameters, we can construct the curves given in Fig. 3 for various values of $T_{s}$.

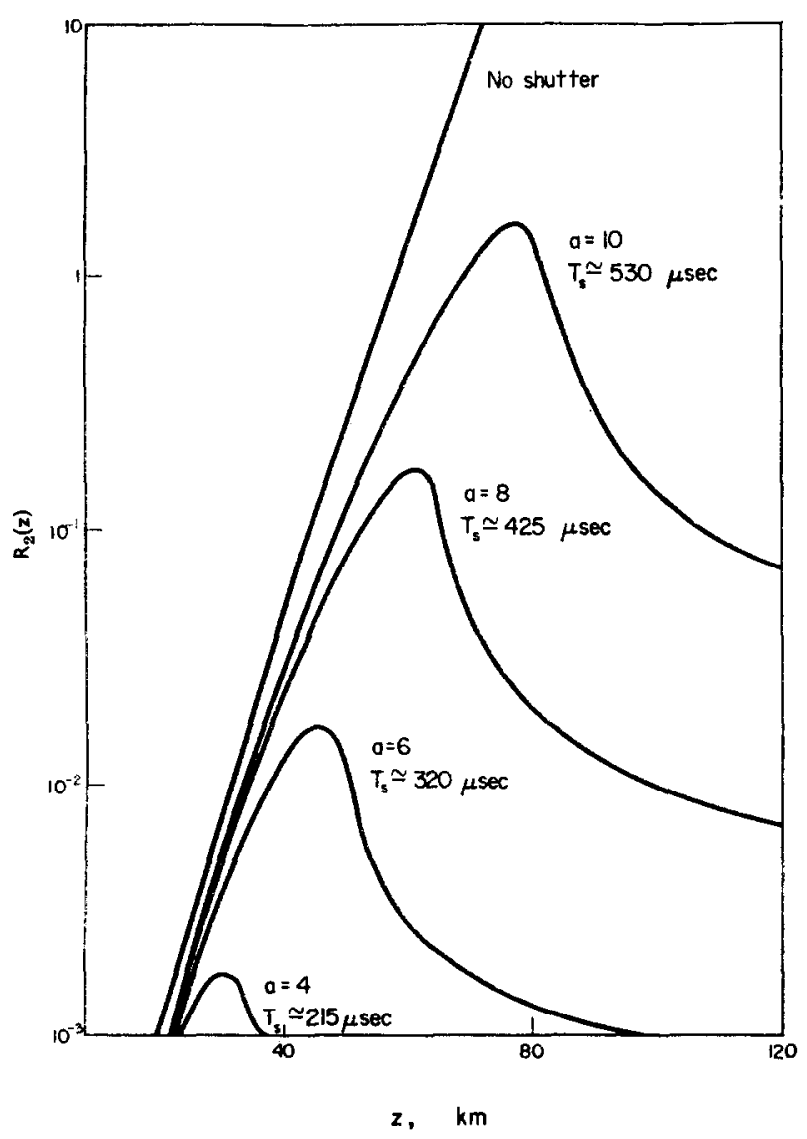

Fig. 3. Ratio of fluorescence backscatter to molecular return $(\beta=1)$.

An inspection of Fig. 3 shows that it is indeed possible to generate a spurious "layer" structure when using a shutter to suppress fluorescence. In particular, for $a=10$ a fairly large peak occurs near $z=76 \mathrm{~km}$. For $\beta=1, R_{2} \simeq 1.7$ for this peak. For $\beta=0 \cdot 1$ (i.e., assume that the fluorescence is emitted into a fairly small cone from the ruby) then $R_{2} \simeq 17$. The presence of aerosols would alter the above results in a manner similar to that discussed in the previous section.

Although a given shutter setting will produce only one peak, it should be kept in mind that, due to the very weak returns from the upper atmosphere, profiles must generally be constructed from the results of hundreds of shots taken over a period of, perhaps, several days. This admits the possibility that slightly different shutter settings might be used for the different nights which would produce multiple peaks in the composite profile. 
We must, however, stress two important points:

(1) To generate a significant (say greater than five percent) "layer" the shutter closing time, $T_{s}$, must be greater than about $375 \mu$ sec for $\beta=1$. This relatively large value for $T_{s}$ is probably not typical of operational optical radar systems. For example, the system used by McCormick et al. has a $T_{s}$ of $250 \mu \sec \left(R_{2} \simeq\right.$ $5 \times 10^{-3}$ at the peak for $\beta=1 ; R_{2} \simeq 5 \times 10^{-2}$ for $\beta=0 \cdot 1$ ).

(2) The "layers" tend to be spread out over several atmospheric scale heights (although it is possible, by assuming a shutter cutoff that is not linear, to "sharpen up" the structure).

The effect of the shutter may easily be determined by misaligning the transmitter and receiver slightly and observing the signal with and without the shutter. This technique was not possible with the University of Maryland system since a single optical path was used for transmitting and receiving. The transmit-receive shutter prevented the PMT from receiving any signal until ruby fluorescence was blocked from entering the atmosphere. Since, as stated above, the cutoff time was $250 \mu \mathrm{sec}$ for the Maryland system it can be seen from Fig. 3 that no detectable (compared to PMT dark current and sky background) noise would be expected. This was verified experimentally by firing fluorescence (only) through the system and observing the PM'T output. It would be possible, by using various shutter speeds, to verify the predictions shown in Fig. 3 for larger values of cut-off time even when using a single optical path system.

\section{Conclusion}

Based upon the preceding remarks it is concluded that, in order to insure that ruby fluorescence does not contaminate the optical radar returns, a shutter cut-off time of less than about $375 \mu \mathrm{sec}$ is required. It is also concluded that backscattered ruby fluorescence will not explain the enhanced returns (about 10 times the molecular value in the 77-82 km region) observed by MaConMrck et al. It should be emphasized however, that before an in situ source may be assumed for the enhanced returns effects such as multiple scattering and possible non-random processes in the detection statistics must be thoroughly investigated.

Acknowledgement-I wish to thank Professors R. T. Bettinger and S. K. Poultney, of the University of Maryland, for fruitful discussions on this subject. This work was supported, in part, by the Advanced Research Projects Agency under contract DAHCl5-68-C-0144.

\section{REFERENCES}

BaIN W. C. and Sandford M. C. W. Kent G. S., Clemesha B. R. and WRight R. W.

McCormick P. D., Poultney S. K., vaN WiJK U., Alley C. O., Bettinger R. T. and Perschy J. A.

McCormick P. D., Silverberg E. C., Poultiney S. K., Van WIJK U., Auley C. O. and Bettinger R. T.

Northend C. A., Honey R. C. and Evans W. E.

SANDFORd M. C. W.

\section{6}

1967

1966

1967

Nature, Lond. 215, 1262.

1966

1967

Nature, Lond. 209, 798.
J. Atmosph. Terr. Phys. 28, 543.

J. Atmosph. Terr. Phys. 29, 169.

Rev. Sci. Instrum. 37, 393.

J. Atmosph. Terr. Phys. 29, 1657. 


\section{ApPendix 1}

\section{Evaluation of a Fluorescence Integral}

In section 3 we derive an expression for the expected count rate, at time $t=2 z / c$, due to fluorescence which involves the integral:

$$
\begin{aligned}
I & =\int_{z_{0} / H}^{z / H} \frac{e^{-x} d x}{x^{2}} \\
H & =\text { atmospheric scale height } \simeq 8 \mathrm{~km} ; \\
z_{0} & =\text { beam overlap altitude } \simeq 2 \mathrm{~km} .
\end{aligned}
$$

We have:

$$
\begin{aligned}
& I=\left[-\frac{e^{-x}}{x}\right]_{z_{0} / H}^{z / H}-\int_{z_{0} / H}^{z / H} \frac{e^{-x}}{x} d x \\
& I=\frac{e^{-z_{0} / H}}{\left(z_{0} / H\right)}-\frac{e^{-z / H}}{(z / H)}-\int_{z_{0} / H}^{\infty} \frac{e^{-x}}{x} d x+\int_{z / H}^{\infty} \frac{e^{-x}}{x} d x \\
& I=\frac{e^{-z_{0} / H}}{\left(z_{0} / H\right)}-\frac{e^{-z / H}}{(z / H)}-E_{1}\left(z_{0} / H\right)+E_{1}(z / H)
\end{aligned}
$$

$$
\left(E_{1}(x)\right. \text { is the exponential integral). }
$$

Now, $z_{0} / H \simeq 0.25$ and, from tables we find that $E_{1}(0.25) \simeq 1.04$. Also:

$$
(z / H) e^{z / H} E_{1}(z / H) \simeq \frac{(z / H)^{2}+2 \cdot 33(z / H)+0 \cdot 25}{(z / H)^{2}+3 \cdot 33(z / H)+1 \cdot 68} \quad 1 \leqslant z / H<\infty .
$$

If we restrict ourselves to altitudes above $48 \mathrm{~km}(z / H \simeq 6)$ we have:

$$
E_{1}(z / H) \simeq \frac{e^{-z / H}}{(z / H)}
$$

Therefore:

$$
I \simeq \frac{e^{-0.25}}{(0 \cdot 25)}-1 \cdot 04 \simeq 2 \quad \text { for } \quad z>48 \mathrm{~km}
$$

